\title{
Desarrollo osteológico de la columna vertebral y del complejo caudal de larvas de Lutjanus guttatus (Perciformes: Lutjanidae) en condiciones de cultivo
}

\author{
Luz Estela Rodríguez-Ibarra*, María Isabel Abdo-de la Parra, Gabriela Aguilar-Zárate, \\ Gabriela Velasco-Blanco \& Leonardo Ibarra-Castro \\ Centro de Investigación en Alimentación y Desarrollo, A.C. Unidad Mazatlán, Av. Sábalo-Cerritos S/N, C.P. 82100, \\ Mazatlán Sinaloa, México; eibarra@ciad.mx, abdo@ciad.mx,gaguilar@ciad.mx,gvelas@ciad.mx, \\ leonardo.ibarra@ciad.mx
}

Recibido 26-V-2014. Corregido 20-VIII-2014. Aceptado 22-IX-2014.

\begin{abstract}
Osteological development of the vertebral column and caudal complex of Lutjanus guttatus (Perciformes: Lutjanidae) larvae under rearing conditions. The spotted rose snapper (Lutjanus guttatus) is an important commercial species in Mexico with good culture potential. The osteological study at early stages in this species is an important tool to confirm normal bone structure and for the detection of malformations that may occur during early development. This study was carried out in order to evaluate and describe the normal osteological development of the vertebral column and caudal complex of this species grown under controlled conditions. For this, a total of 540 larvae of L. guttatus, between 2.1 and $17.5 \mathrm{~mm}$ of total length (TL), were cultured during 36 days; culture conditions were $28^{\circ} \mathrm{C}, 5.74 \mathrm{mg} / \mathrm{L}$ oxygen and $32.2 \mathrm{ups}$ salinity with standard feeding rates. To detect growth changes, a sample of 15 organisms was daily taken from day one until day 36 of post-hatch (DPH). Samples were processed following standard techniques of clearing, and cartilage (alcian blue) and bone staining (alizarin red). Results showed that the vertebral column is composed of ten vertebrae in the abdominal region, and 14 vertebrae including the urostyle in the caudal region. The development of the axial skeleton starts with the neural arches and haemal arches at $3.8 \mathrm{~mm}$ TL. Caudal elements such as the hypurals and parahypural began to develop at $4.1 \mathrm{~mm}$ TL. Pre-flexion and flexion of the notochord and the formation of all hypurals were observed between 5.3 and $5.8 \mathrm{~mm}$ TL. Ossification of the vertebrae in the abdominal region and in some neural arches initiated at $9.5 \mathrm{~mm}$ TL. In the caudal region, all the neural and haemal arches ossified at $10.2 \mathrm{~mm}$ TL. All the abdominal vertebrae and their respective neural arches and parapophyses ossified at $11.2 \mathrm{~mm}$ TL, while the elements of the caudal complex that ossified were the hypurals, parahypurals and modified haemal spines. All caudal fin rays, 12 neural spines and 3 haemal arches were ossified by $15.5 \mathrm{~mm}$. The complete ossification process of this specie under laboratory culture conditions was observed when larvae reached $17.3 \mathrm{~mm}$ TL on $36 \mathrm{DPH}$. Detailed analysis of the osteological structures will allow a reference description to evaluate and detect malformations that may occur during the larval culture of the spotted rose snapper. Rev. Biol. Trop. 63 (1): 155-164. Epub 2015 March 01.
\end{abstract}

Key words: Lutjanus guttatus, larvae, osteology, vertebral column, caudal complex.

El pargo flamenco Lutjanus guttatus (Steindachner, 1869), es una especie que se distribuye a lo largo de las costas del Pacífico Oriental desde el Golfo de California hasta Perú (Fischer et al.,1995; Thomson, Findley, \& Kerstitch, 2000). Es considerada de gran potencial en la maricultura en México y América Latina, y muy apreciada en el mercado nacional por la calidad y sabor de su carne, que es vendida a buen precio (7 USD $/ \mathrm{kg}$ ) (Abdo-de la Parra, \& Rodríguez-Ibarra, 2011; Abdo-de la Parra, Rodríguez-Ibarra, Velasco-Blanco, García-Aguilar, \& González-Rodríguez, 2011; García-Aguilar, \& González-Rodríguez, 2011; García-Ortega et al., 2005).

Esta especie junto con sus congéneres, representan un recurso pesquero muy importante para los pescadores de la región del 
Pacífico Oriental, pero la constante captura ha dado lugar a una elevada tasa de explotación, lo cual ha puesto en peligro su disponibilidad. Por lo tanto, para amortiguar un poco este problema y dada la demanda que tiene esta especie en el mercado, se ha visualizado una alternativa tecnológicamente viable que es su cultivo en jaulas flotantes costeras (Castillo-Vargasmachuca, Ponce-Palafox, Chávez-Ortiz, \& ArredondoFigueroa, 2007), pero para ello, se requiere de la producción intensiva de semillas de alta calidad, con el fin de cubrir estas necesidades.

Sin embargo, durante años en diversos ciclos de cultivo de esta especie en las instalaciones de la unidad Mazatlán del Centro de Investigación en Alimentación y Desarrollo donde se cultiva esta especie a escala masiva, se han observado malformaciones esqueléticas en la columna vertebral (Rodríguez-Ibarra, Aguilar-Zárate, Velasco-Blanco, \& Ibarra-Castro, 2013a). La lordosis se presenta de forma constante como en un $25 \%$ de los organismos cultivados, aproximadamente a los 30 días después de la eclosión (DDE) (obs. pers.), lo que requiere una selección manual de los organismos para separarlos del resto. Es de suma importancia hacer este tipo de selección, ya que puede inferir en los rendimientos de los criaderos de peces, porque los organismos pueden tener problemas tales como habilidad para nadar, en su índice de conversión, en el porcentaje de crecimiento, supervivencia, estrés, infecciones por patógenos, entre otros. Asimismo, durante la etapa de comercialización, es difícil que los consumidores acepten organismos deformes, lo cual conlleva a una pérdida económica importante para estas instalaciones de cultivo (Boglione, Gagliardi, Scardi, \& Cataudella, 2001; Fernández et al., 2008; Lewis-McCrea, Lall, \& Eckhard, 2004).

Estas deformidades comúnmente son asociadas a los sistemas de producción intensiva en criaderos y representan un grave problema para la acuicultura (Fraser, Anderson, \& de Nys, 2004; Hernández, Santinón, Sánchez, \& Domitrovic, 2013). El estudio temprano del desarrollo de los peces cultivados, permite el conocimiento de los caracteres merísticos y morfológicos del sistema osteológico de la columna vertebral y el complejo caudal, lo cual da la oportunidad de detectar y eliminar oportunamente a los peces que presenten malformaciones esqueléticas (Hernández et al., 2013; Çoban, Suzer, Kamaci, Saka, \& Firat, 2009; Koumoundouros, Divanach, \& Kentouri 2001a).

A la fecha no existe información disponible del desarrollo osteológico de la columna vertebral y del complejo caudal del pargo flamenco Lutjanus guttatus. Se han realizado estudios similares en especies pertenecientes a la familia Sparidae (Koumoundouros et al., 1997; Koumoundouros, Divanach, \& Kentouri, 1999) y algunos peces planos (Gavaia, Dinis, \& Cancela, 2002; Lewis-McCrea et al., 2004), pero éste sería el primer trabajo que se hace con un miembro de la familia Lutjanidae en etapa larvaria. El objetivo del presente trabajo fue describir a detalle el desarrollo osteológico normal de la columna vertebral y del complejo caudal de las larvas de L. guttatus; lo cual, servirá de base para poder evaluar en futuras investigaciones la calidad de las larvas, y detectar las posibles malformaciones que se puedan presentar durante la fase del cultivo larvario.

\section{MATERIALES Y MÉTODOS}

Cultivo larvario: El cultivo larvario de L. guttatus se realizó en el área de larvicultura del Centro de Investigación en Alimentación y Desarrollo (CIAD) en la unidad Mazatlán, donde los huevos se obtuvieron del lote de reproductores en cautiverio que se encuentran confinados en tanques de $50 \mathrm{~m}^{3}$. Los huevos fueron sembrados en tres tanques de $1 \mathrm{~m}^{3}$ en una densidad de 42 huevos/L. El cultivo larvario se llevó a cabo a una temperatura promedio de $28^{\circ} \mathrm{C}, 5.74 \mathrm{mg} / \mathrm{L}$ de $\mathrm{O}_{2}$ y salinidad de 32.2UPS, utilizando el protocolo de Abdo-de la Parra et al. (2010) con algunas modificaciones.

Las larvas fueron cultivadas usando la técnica de agua verde (Abdo-de la Parra et al., 2010; Abdo-de la Parra, \& Rodríguez-Ibarra, 2011; García-Ortega, 2009; Rodríguez-Ibarra et al., 2013b), donde a partir del primer día y 
hasta el 13 (DDE) se añadió a los tanques de cultivo, con una frecuencia de una vez al día, una mezcla de las microalgas Nannochloropsis oculata (500000 células $/ \mathrm{mL})$ e Isochrysis galbana. (50000 células/mL). Del segundo hasta el sexto DDE se añadieron 10 rotíferos $/ \mathrm{mL}$ (Brachionus rotundiformis) aumentando a 20 rotíferos/mL a partir del 7 hasta el $15 \mathrm{DDE}$; y finalmente se redujo a 10 rotíferos $/ \mathrm{mL}$ cuando se añadieron los nauplios de Artemia sp. (0.5/ $\mathrm{mL})$ los cuales se incrementaron gradualmente hasta 4 rotíferos $/ \mathrm{mL}$. Asimismo, a partir de los dos DDE hasta el 15 DDE se agregó una concentración de 0.5 copépodos $/ \mathrm{mL}$ de la especie Tisbe monozota. El alimento vivo presente en cada tanque se contó diariamente para ajustarlo a la cantidad requerida, la proporción suministrada por día de los rotíferos y de los nauplios de Artemia sp. fue $50 \%$ por la mañana, $25 \%$ a medio día y $25 \%$ por la tarde.

El destete, o el cambio del alimento vivo al artificial se realizó a partir del 24 DDE, con una dieta comercial microparticulada de 0.5 a $0.8 \mathrm{~mm}$ (Skretting ${ }^{\circledR}$, Fontaine les Vervins, France), con una reducción gradual del número de nauplios de Artemia sp., hasta sustituirlos completamente por la dieta artificial.

Las larvas se cultivaron utilizando luz continua las $24 \mathrm{~h}$ durante los primeros $15 \mathrm{DDE}$. A partir del $16 \mathrm{DDE}$, el fotoperiodo se redujo a 12:12 h de luz: oscuridad. Del uno al 13 DDE se hizo un recambio de agua de un $30 \%$ (aproximadamente 180L) antes de abrirse el flujo, para mantener una buena calidad del agua. A los 14 DDE se suministró flujo continuo para obtener un recambió de 0.2 volúmenes de agua al día y se aumentó gradualmente hasta recambiar tres volúmenes al día. El flujo de aire se mantuvo a $0.5 \mathrm{~L} / \mathrm{min}$ del uno a $15 \mathrm{DDE}$ y se incrementó hasta alcanzar $2 \mathrm{~L} / \mathrm{min}$ a partir del 25 DDE.

Recolecta de muestras y técnica de tinción de cartílagos y huesos: Para la descripción del desarrollo osteológico de larvas de $L$. guttatus, se evaluaron larvas que presentaron desarrollo normal y fue preciso tomar una muestra de 15 organismos (cinco de cada tanque) diariamente, a partir del día uno y hasta el $36 \mathrm{DDE}$ a los cuales se les registró su longitud total (LT) con un vernier digital con precisión de $0.01 \pm 0.03 \mathrm{~mm}$. Posteriormente, se sacrificaron con una sobredosis $(0.1 \mathrm{ml} / \mathrm{L})$ del anestésico 2-fenoxietanol (Sigma Aldrich), para someterlos al protocolo de tinción de cartílago y hueso (Potthoff, 1984) que a continuación se describe:

- Fijación por 24 horas en formol (Hycel) al $4 \%$ en buffer fosfato.

- Deshidratación por 24 horas en etanol al $50 \%$ (Fisher) y transferencia a etanol $100 \%$ otras 24 horas.

- Tinción de cartílago de uno a dos días (de acuerdo al tamaño) utilizando $(70 \mathrm{~mL}$ etanol absoluto, 30mL ácido acético (JT Baker) y 20mg de azul aciano (Fluka).

- Neutralización 12 horas con borato de sodio $\left(\mathrm{Na}_{2} \mathrm{~B}_{4} \mathrm{O}_{7}\right)$ (Fisher) saturado.

- Blanqueamiento 40 minutos en $15 \mathrm{~mL}$ de peróxido $\left(\mathrm{H}_{2} \mathrm{O}_{2}\right)$ (Fermont) al $3 \%$ y $85 \mathrm{~mL}$ hidróxido de potasio $(\mathrm{KOH})$ (Fisher) al $1 \%$; en organismos pequeños se utilizó por unos cuantos segundos hasta observar que no se formaran burbujas en el organismo.

- Aclaramiento con una solución de $35 \mathrm{~mL}$ $\mathrm{Na}_{2} \mathrm{~B}_{4} \mathrm{O}_{7}$ saturado, $65 \mathrm{~mL}$ agua destilada y tripsina 1mg (Sigma Aldrich). El tiempo que duró el organismo fue directamente proporcional al tiempo de digestión de la enzima sobre el músculo, y se detuvo la reacción cuando el organismo se transparentó.

- Tinción de hueso, se utilizó $100 \mathrm{~mL} \mathrm{KOH}$ y $10 \mathrm{mg}$ rojo alizarina (Sigma Aldrich) de uno a cuatro días, de acuerdo al tamaño del organismo.

- Transparentar con una solución de $35 \mathrm{~mL}$ $\mathrm{Na}_{2} \mathrm{~B}_{4} \mathrm{O}_{7}$ saturado, $65 \mathrm{~mL}$ agua destilada y tripsina de uno a dos días, hasta eliminar los restos de color y que terminara de digerir el músculo que haya quedado en el organismo.

- Decoloración con $\mathrm{KOH} \mathrm{1 \% .} \mathrm{El} \mathrm{tiempo} \mathrm{fue}$ directamente proporcional al tamaño del organismo. Se hicieron recambios de la 
solución cada 10 días o más si requirió de más tiempo para decolorar.

- Preservación, se utilizó glicerina a diferentes concentraciones, primero al $30 \%$, después al $60 \%$ en $\mathrm{KOH}$, y finalmente al $100 \%$.

Después de haber concluido el proceso de tinción hasta preservación de los organismos, se llevó a cabo el análisis de los mismos, el cual consistió en observar las muestras de las larvas a través de un microscopio (Olympus CX31) (cuando midieron menos de $2.5 \mathrm{~mm}$ ) y un estereoscópico (Olympus SZ) (cuando midieron más de $2.5 \mathrm{~mm}$ ). Ambos equipados con una cámara (Olympus SP-350) para tomar fotos de la columna vertebral y de la parte caudal de los organismos, en cada uno de los estadios de desarrollo, con el fin de describir las de estructuras óseas y cartilaginosas. Los elementos osteológicos se describieron de acuerdo a la terminología utilizada por Lagler, Bardach, Miller \& May Passino (1990), Koumoundouros et al. (1997), Koumoundouros et al. (1999), Koumoundouros et al. (2001a), Koumoundouros, Sfakianakis, Maingot, Divanach \& Kentouri (2001b), Boglione et al. (2001), Gavaia et al. (2002), Meunier \& Ramzu (2006), Gállego (2008), Bensimon-Brito, Cancela, Huysseune \& Witten (2010) y Laggis, Sfakianakis, Divanach \& Kentouri (2010).

\section{RESULTADOS}

Columna vertebral y complejo caudal: En el presente trabajo fueron procesados un total de 540 organismos con un rango de tallas de 2.1 a $17.3 \mathrm{~mm}$ (LT), los cuales fueron cultivados hasta el 36 DDE que es cuando los organismos se cosechan para pasar a la etapa de pre-engorda. Después de analizar cada uno de los organismos transparentados y teñidos, se determinó que la columna vertebral de larvas de L. guttatus está compuesta por 21 vértebras centrales, 21 arcos y espinas neurales, 11 arcos y espinas hemales, ocho costillas ventrales y cinco parapófisis. Asimismo, el complejo caudal se compone de dos centros preurales (vértebras), urostilo, tres epurales, cinco hipurales, un parahipural, un arco y espina neural, dos arcos y espinas hemales y un arco neural especializado (Fig. 1).

La columna vertebral se divide principalmente en dos regiones, la abdominal y la caudal, diez vértebras son abdominales y 14 caudales incluyendo el urostilo. Cada vértebra abdominal se compone por su parte dorsal de un arco y espina neural, mientras que por la parte ventral, cinco vertebras están equipadas con parapófisis (lugar donde se forman algunas costillas ventrales). Las vértebras caudales están equipadas con arcos y espinas neurales dorsalmente $\mathrm{y}$, por arcos y espinas hemales ventralmente. La última espina neural (espina

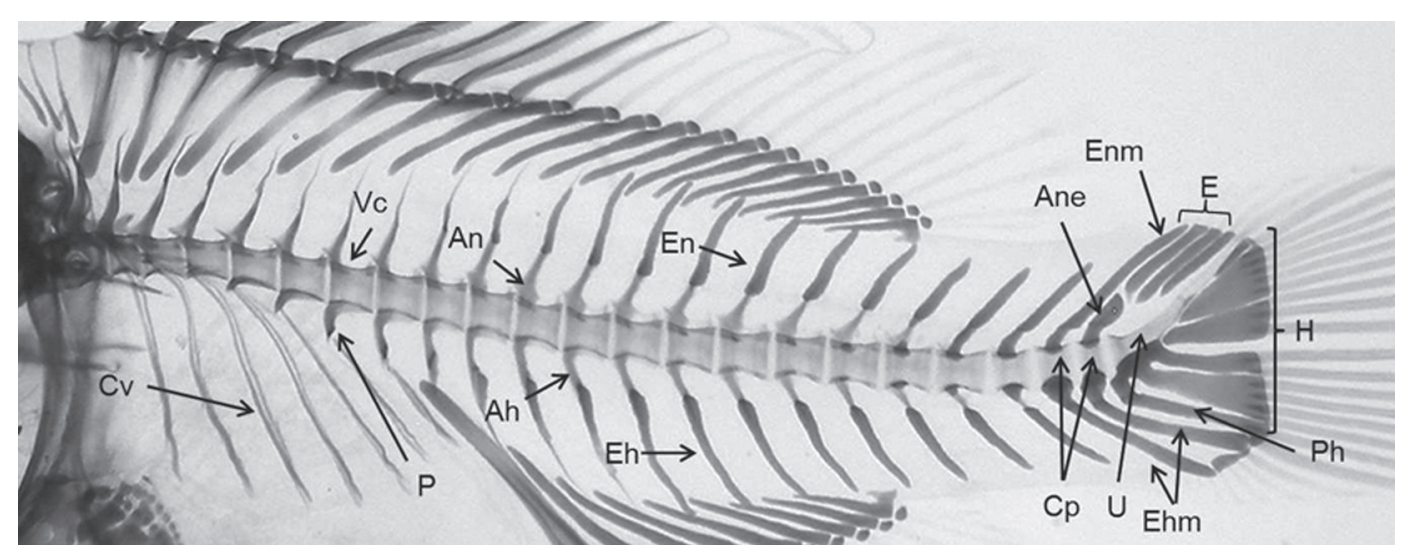

Fig. 1. Estructuras osteológicas que componen la columna vertebral y el complejo caudal de larvas de pargo flamenco. Fig. 1. Osteological structures forming the spine and the caudal complex of spotted rose snapper larvae. 
neural modificada) y las dos hemales (espina hemal modificada), se prolongan para dar soporte a los radios de la aleta caudal (Fig. 1).

En el cuadro 1 se enlistan los nombres y abreviaturas de los elementos que componen la columna vertebral y el complejo caudal.

\section{CUADRO}

Elementos del esqueleto de Lutjanus guttatus y sus abreviaciones

TABLE 1

Skeletal elements and their abbreviations of Lutjanus guttatus

\begin{tabular}{llc} 
& \multicolumn{1}{c}{ Elemento del esqueleto } & Abreviatura \\
Columna vertebral & Vértebras centrales & $\mathrm{Vc}$ \\
& Arcos neurales & $\mathrm{An}$ \\
& Espinas neurales & $\mathrm{En}$ \\
& Costillas ventrales & $\mathrm{CV}$ \\
& Parapófisis & $\mathrm{P}$ \\
& Arco hemal & $\mathrm{Ah}$ \\
Complejo caudal & Espina hemal & $\mathrm{Eh}$ \\
& Epurales & $\mathrm{E}$ \\
& Hipurales & $\mathrm{H}$ \\
& Parahipural & $\mathrm{Ph}$ \\
& Espina neural modificada & $\mathrm{Enm}$ \\
& Espina hemal modificada & $\mathrm{Ehm}$ \\
& Arco neural especializado & $\mathrm{Ane}$ \\
& Centros preurales & $\mathrm{Cp}$ \\
& Urostilo & $\mathrm{U}$ \\
Lepidotriquia & $\mathrm{L}$ \\
Dermatotriquia & $\mathrm{D}$ \\
Accesorio cartilaginoso & $\mathrm{Ac}$ \\
\hline
\end{tabular}

Desarrollo de las estructuras de la columna vertebral y del complejo caudal durante el cultivo larvario: Durante los primeros días de desarrollo de las larvas no se observó presencia de elementos vertebrales, siendo el notocordio la única estructura presente de la suspensión axial.

Se observó la presencia de estructuras cartilaginosas en la columna vertebral a partir del 12 DDE cuando la larva registró una LT promedio de $3.8 \mathrm{~mm}$. Se observó la aparición de los arcos neurales del uno al cuatro y del 10 al 13, y los arcos hemales del uno al cinco (Fig. 2a).

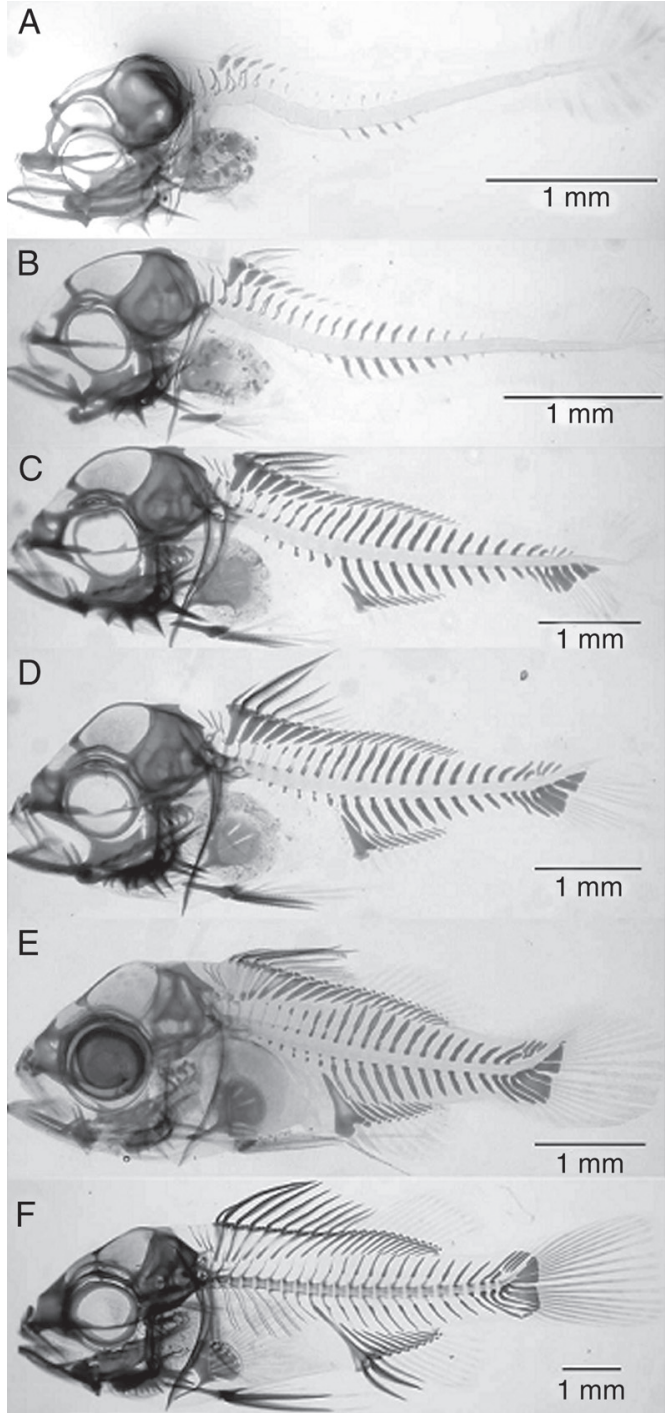

Fig. 2. Desarrollo normal de la columna vertebral y del complejo caudal del pargo flamenco en diferentes etapas de desarrollo bajo condiciones de cultivo; A) $3.9 \mathrm{~mm} \mathrm{LT}$, B) $4.1 \mathrm{~mm} \mathrm{LT}$, C) $5.3 \mathrm{~mm} \mathrm{LT}$, D) $5.8 \mathrm{~mm} \mathrm{LT}$, E) $6.3 \mathrm{~mm} \mathrm{LT}$ y F) $11.2 \mathrm{~mm} \mathrm{LT}$.

Fig. 2. Normal development of the vertebral column and caudal complex of the spotted rose snapper at different development stages under culture conditions; A) $3.9 \mathrm{~mm}$ TL, B) $4.1 \mathrm{~mm} \mathrm{TL}$, C) $5.3 \mathrm{~mm} \mathrm{TL}$, D) $5.8 \mathrm{~mm} \mathrm{TL}$, E) $6.3 \mathrm{~mm}$ $\mathrm{TL}$ and F) $11.2 \mathrm{~mm} \mathrm{TL}$.

Estos arcos, se desarrollan inicialmente como dos brotes de cada lado del notocordio, los cuales posteriormente se prolongan y se unen entre sí, para dar origen a cada uno de los arcos. 
A los $14 \mathrm{DDE}$ (4.1mm LT), inició la formación de los elementos que conformaron el complejo caudal, se observó la presencia de los hipurales uno y dos y el parahipural. También inició la formación de lo que daría origen a los primeros parapófisis del seis al ocho, en esta etapa se han formado los arcos neurales uno al $17 \mathrm{y}$ los hemales uno al ocho y es poco visible el nueve (Fig. 2b).

A los $16 \mathrm{DDE}$ (5.3mm LT), ya se observó la presencia de la mayoría de los elementos cartilaginosos de la columna vertebral; en el complejo caudal faltan por formarse algunos y el notocordio sigue recto. En la columna vertebral ya se han formado los 21 arcos y espinas neurales y los $11 \operatorname{arcos}$ y espinas hemales. El complejo caudal ya se observa por su parte dorsal, el arco de la espina neural modificada y el arco neural especializado y los tres epurales. Por la parte ventral, se ha formado una de las dos espinas hemales modificadas y solo el arco de una de ellas, así como el parahipural, y los hipurales uno al cuatro, y se observó la presencia de los radios caudales que se encuentran en la parte distal de los hipurales (Fig. 2c). Cabe señalar que el parahipural y los cuatro hipurales, se encuentran separados entre ellos.

A los 18 DDE (5.8mm LT) se empieza a flexionar el notocordio (pre-flexión) (Fig. 2d), y en el día 22 DE ya se encuentra flexionada completamente y las larvas presentan una LT promedio de $6.3 \mathrm{~mm}$. También se observó la presencia del hipural cinco y todos los radios caudales ya se encuentran presentes, sin estar calcificados, y ya se observa la unión de sus bases de los hipurales uno y dos con el parahipural (Fig. 2e).

El día $24 \mathrm{DE}$ (9.5mm LT), se observó osificación de las primeras cuatro vértebras centrales de la región abdominal (en dirección a caudal) y algunos arcos neurales (del uno al siete). Asimismo, se observó el desarrollo de las tres primeras costillas ventrales. Entre el día 26 y 27 DE (10.2mm LT), se observó osificación en todas las vértebras centrales de la región abdominal y caudal, además de los arcos neurales uno al 21 y los nueve arcos hemales, al igual que las bases de los radios de la aleta caudal principal, y se observó la presencia de las ocho costillas ventrales.

A los 28 DDE (11.2mm LT), se observó la osificación de casi toda la región abdominal (vértebras abdominales con sus respectivos arcos neurales y parapófisis), así como caudal (centros preurales y urostilo, los hipurales, parahipurales y en las espinas hemales modificadas). A los 32 DDE (15.5mm LT), se completó la osificación de los radios de la aleta caudal, además de 12 de las 21 espinas neurales y tres hemales. La parte distal de las hipurales, epurales y espinas hemales y neurales del complejo caudal, permaneció cartilaginosa y es justo donde se articulan con los radios de la aleta caudal (Fig. 2f).

Para el 36 DDE (17.3mm LT) último día del experimento, la mayoría de las estructuras de la columna vertebral y del complejo caudal presentaron osificación a excepción de las espinas neurales 14 a 21 y hemales 5 a 11 $\mathrm{y}$ las epurales.

Conforme la parte final de la columna vertebral se va haciendo curva en sentido dorsal, debido a la flexión del notocordio, los elementos hipurales sufren una separación entre ellos, quedando en la parte baja los hipurales uno y dos, y en la parte superior del tres al cinco. Los radios principales de la aleta caudal también llamada lepidotriquia (L) se conformó por 18 radios, nueve en la parte inferior, los cuales se encuentran distribuidos y apoyados de la siguiente manera en las estructuras del complejo caudal: En la parte inferior, el radio uno y dos se apoyan en la segunda espina hemal modificada (Ehm), el tres y parte del cuatro en la parahipural $(\mathrm{Ph})$, parte del cuatro al siete en el hipural uno (H1), y el radio ocho y nueve en el hipural dos (H2). El número de radios en la parte superior fue de nueve y se distribuyen de la siguiente manera: uno, dos y parte del tres en el hipural tres (H3), parte del tres al ocho en el hipural cuatro (H4) y en el hipural cinco (H5) se apoya el radio número nueve. La aleta caudal también cuenta con radios blandos que son llamados dermatotriquia (D), y se encuentran distribuidos en los extremos de los radios principales; en la parte dorsal se encuentran 
distribuidos en los epurales (E) y en la parte ventral se encuentra sobre la primera espina hemal modificada (Ehm) y sobre una estructura llamada accesorio cartilaginoso (Fig. 3).

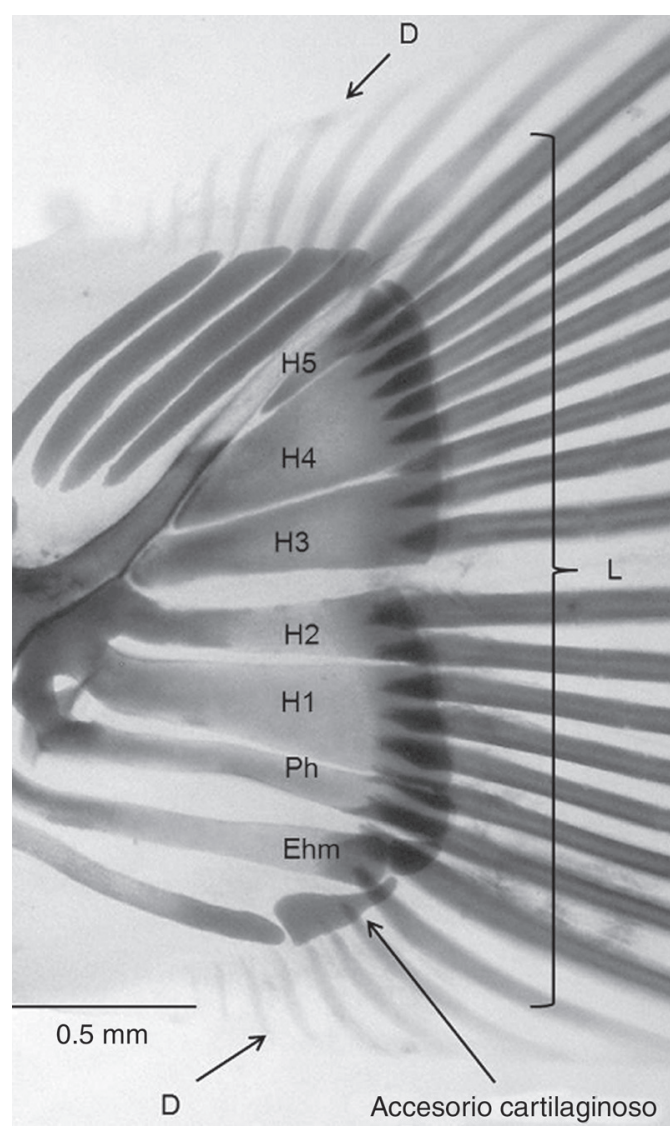

Fig. 3. Distribución de los radios de la aleta caudal principal del pargo flamenco en algunas estructuras del complejo caudal.

Fig. 3. Distribution of the principal caudal fin rays in some structures of the caudal complex of the spotted rose snapper.

\section{DISCUSIÓN}

En especies cultivables y con una alta demanda económica como Sparus aurata, Solea senegalensis, Lates calcarifer e Hippoglossus hippoglossus y en el caso particular del Lutjanus guttatus, se han manifestado malformaciones del esqueleto de dichos organismos desde edades tempranas (Fernández et al.,
2008; Fraser et al., 2004; Gavaia et al., 2002; Koumoundouros et al., 1997; Lewis-McCrea et al., 2004; Rodríguez-Ibarra et al., 2013a). En general, estas deformidades morfológicas influyen directamente en el bajo rendimiento de los criaderos de peces, reflejado en baja tasa de supervivencia, poco crecimiento, escasa competencia por el alimento y un aumento considerable a estrés y enfermedad. Asimismo, estas anomalías de la columna vertebral afectan significativamente la forma y el tamaño de los organismos, lo cual se ve reflejado en una baja demanda y una depreciación del producto (Boglione et al., 2001; Lewis-McCrea et al., 2004; Fernández et al., 2008).

En el presente trabajo se describió el desarrollo osteológico de la columna vertebral y el complejo caudal del pargo flamenco $L$. guttatus, durante los primeros estadios de vida bajo condiciones controladas de cultivo. Sin embargo, existen diversas causas que provocan alteraciones en el desarrollo normal del esqueleto en peces cultivados, como factores ambientales, genéticos, sistemas intensivos de cría y en menor cantidad por el efecto de contaminantes (Fernández et al. 2008; Hernández et al., 2013). Por lo tanto, el conocimiento del desarrollo osteológico normal de esta especie de pargo, es una herramienta, la cual permitirá registrar los cambios progresivos de cada uno de los elementos esqueléticos que conforman el organismo, y detectar precozmente cuándo y en qué momento se presentan condiciones de cultivo inadecuadas (Fraser et al., 2004; Hernández et al., 2013).

El desarrollo de las estructuras que componen el sistema óseo del pargo inicia después de la eclosión, al igual que otras especies como las pertenecientes a la familia Sparidae (Koumoundouros et al., 1997, 2001b) o algunos peces planos (Wagemans, Focant, \& Vandewalle, 1998; Wagemans, \& Vandewalle, 1999); el momento de la aparición de las mismas dependen de diversos procesos biológicos propios de la especie, entre ellos está la temperatura del agua de cultivo. Durante el cultivo larvario se registró una temperatura del agua $28^{\circ} \mathrm{C}$ y de acuerdo a Çoban et al. (2009), este es uno de 
los factores que influyen directamente en el desarrollo osteológico de las especies, donde el crecimiento es relativamente más rápido y por consiguiente la aparición de las estructuras se hacen presentes en un periodo de tiempo más corto. En el presente trabajo si bien, antes de los 12 DDE no se había formado ninguna estructura, a partir de esta edad fueron apareciendo la mayoría de ellas en periodo de 10 días (12 al 22 DDE). Del 22 al 28 DDE, se observó un crecimiento significativo de los organismos, alcanzando tallas promedio de casi el doble de LT. Este factor de la temperatura, no se presenta en especies como Sparus aurata, Pagrus pagrus, Diplodus sargus, donde su cultivo larvario se desarrolla a temperaturas más bajas entre 19 y $23^{\circ} \mathrm{C}$, (Castelló-Orvay, 1993; Koumoundouros et al., 1999, 2001a), y el desarrollo de las estructuras esqueléticas es más lento, lo que permite observar y registrar con detenimiento la aparición de la mayoría de ellas.

El complejo caudal o uróforo, es una estructura que juega un papel sumamente importante en un pez, como es la natación, y particularmente compleja ya que durante el desarrollo de los organismos ocurren una serie de modificaciones muy importantes. Durante las primeras fases del desarrollo osteológico de los peces y en el caso particular de esta especie, se observó que la parte final de la columna vertebral se curva en sentido dorsal y los arcos y las espinas neurales reducen su tamaño hasta dejar unas pequeñas espinas aisladas llamadas epurales; mientras que las hemales lo aumentan, debido a que las dorsales tienen menos superficie de inserción que las ventrales. Gállego (2008) menciona que existen dos tipos de complejos caudales, el de las especies primitivas y el de las más evolucionadas; donde las primeras conservan sus cinco hipurales, mientras que en el segundo se presentan fusiones entre ellos. Esta especie es considerada como primitiva de acuerdo a Gállego (2008) y a Koumoundouros et al. (2001b), ya que no presenta este tipo de fusión entre hipurales, y además conserva todas sus estructuras. En las primeras etapas de formación, las hipurales uno y dos y el parahipual, se encontraron unidas en su base cartilaginosa, pero posteriormente se fueron uniendo solo de sus bases, manteniéndose bien delimitadas entre cada una de ellas. Es importante mencionar que, los hipurales en algunos osteíctios (pargos y espáridos) se distribuyen durante su desarrollo en la parte ventral y dorsal de la aleta caudal, pero en su origen son estructuras hemales o ventrales de las vértebras. Si bien, la aleta caudal de estos peces presentan un aspecto simétrico (aleta homocerca), internamente es de forma asimétrica, debido a todas las modificaciones que se presentan durante los primeros estadios de desarrollo y su aspecto es como una aleta heterocerca parecida a la de los condrictios (Gállego, 2008).

El contar con la descripción del desarrollo osteológico normal de esta especie, será la base para trabajos futuros en los cuales se pueda evaluar el efecto de diversos protocolos de alimentación, $\mathrm{y}$ factores abióticos como temperatura y salinidad que puedan incidir en las malformaciones de las larvas; todo esto con la finalidad de optimizar la larvicultura de esta especie y aumentar el número de juveniles de calidad producidos en cautiverio.

Se concluye que, el conocimiento del desarrollo osteológico de la columna vertebral y del complejo caudal de las larvas de L. guttatus, es una herramienta útil para estudiar los procesos detallados de la ontogenia de la especie y poder detectar y descartar aquellos que presenten malformaciones esqueléticas durante el cultivo.

\section{AGRADECIMIENTOS}

Los autores agradecen a V. Williams de Calvario por la traducción del resumen y la asistencia técnica de Juan Huerta, Rosendo Valdivia y Jesús Tirado. Esta investigación fue financiada por el proyecto FORDECyT 173714 bajo la responsabilidad de M.I. Abdo-de la Parra.

\section{RESUMEN}

El pargo flamenco (Lutjanus guttatus) es una especie de importancia comercial en México con un gran potencial para su cultivo. El estudio osteológico en estadios tempranos de esta especie bajo condiciones controladas, 
es una herramienta importante para el conocimiento de su estructura ósea normal y poder detectar las malformaciones que se puedan presentar. El objetivo del presente trabajo se realizó para conocer y describir el desarrollo osteológico normal de la columna vertebral y el complejo caudal de 540 larvas de 2.1 a $17.5 \mathrm{~mm}$ de longitud total (LT) bajo condiciones de cultivo a $28^{\circ} \mathrm{C}, 5.74 \mathrm{mg} / \mathrm{L}$ de oxígeno y 32.2UPS de salinidad. Diariamente se tomó una muestra de 15 organismos desde el día uno hasta el 36 después de la eclosión (DDE) y se procesaron con las técnicas de clareado y tinción de cartílago (azul aciano) y hueso (rojo alizarina) para llevar a cabo la descripción de las estructuras. La columna vertebral se divide en región abdominal con diez vértebras y región caudal compuesta por 14 vértebras incluido el urostilo. El desarrollo del esqueleto axial inicia con la formación de los arcos neurales y hemales a los $3.8 \mathrm{~mm}$ de LT. A los $4.1 \mathrm{~mm}$ de LT empieza la formación de los hipurales y parahipural que son elementos caudales. Entre los 5.3 y $5.8 \mathrm{~mm}$ de LT se observó en pre-flexión y flexión del notocordio y la formación de todos los hipurales. La osificación de las vértebras en la región abdominal y en algunos arcos neurales inició a los $9.5 \mathrm{~mm}$ de LT. A los $10.2 \mathrm{~mm}$ de LT se osificó la región caudal y todos los arcos neurales y hemales. A los $11.2 \mathrm{~mm}$ LT se osificaron todas las vértebras abdominales con sus respectivos arcos neurales y los parapófisis, mientras que los elementos del esqueleto caudal que se osificaron fueron los hipurales, parahipurales y las espinas hemales modificadas. A los $15.5 \mathrm{~mm}$ de LT se osificaron los radios de la aleta caudal y 12 espinas neurales y 3 hemales. El proceso de osificación de las larvas de esta especie en condiciones de cultivo se completó a los $17.3 \mathrm{~mm}$ LT o 36 DDE. El análisis detallado de las estructuras osteológicas, permitirá una descripción de referencia para evaluar y detectar las malformaciones que se puedan presentar durante el cultivo larvario.

Palabras clave: Lutjanus guttatus, larva, osteología, columna vertebral, complejo caudal.

\section{REFERENCIAS}

Abdo-de la Parra, M. I., Rodríguez-Ibarra, L. E., CampilloMartínez, F., Velasco-Blanco, G., García-Aguilar, G., Álvarez-Lajonchère García, L. S., \& VoltolinaLobina, D. (2010). Efecto de la densidad de siembra sobre el crecimiento y supervivencia larval del pargo lunarejo (Lutjanus guttatus). Revista de Biología Marina y Oceanografia, 45, 141-146.

Abdo-de la Parra, M. I., Rodríguez-Ibarra, L. E., VelascoBlanco, G., García-Aguilar, N., \& González-Rodríguez, B. (2011). Evaluación del efecto de diferentes salinidades sobre la incubación de huevos y eclosión de larvas del pargo flamenco Lutjanus guttatus. Ciencia Pesquera, 19(1), 29-34.

Abdo-de la Parra, M. I., \& Rodríguez-Ibarra, L. E. (2011). Cultivo larvario y requerimientos nutricionales del pargo flamenco. Saarbrücken: Academic Publishing Gmbh \& Co. KG.

Bensimon-Brito, A., Cancela, M. L., Huysseune, A., \& Witten, P. E. (2010). The zebrafish (Danio rerio) caudal complex -a model to study vertebral body fusion. Journal of Applied Ichthyology, 26, 235-238.

Boglione, C., Gagliardi, F., Scardi, M., \& Cataudella, S. (2001). Skeletal descriptors and quality assessment in larvae and post-larvae of wild caught and hatcheryreared gilthead sea bream (Sparus aurata L. 1758). Aquaculture, 192, 1-22.

Castelló-Orvay, F. (1993). Acuicultura marina: Fundamentos biológicos y tecnología de la producción. Barcelona: Universitat de Barcelona.

Castillo-Vargasmachuca, S., Ponce-Palafox, J. T., ChávezOrtíz, E., \& Arredondo-Figueroa, J. L. (2007). Effect of the initial stocking body weight on growth of spotted rose snapper Lutjanus guttatus (Steindachner, 1869) in marine floating cages. Revista de Biología Marina y Oceanografia, 42(3), 261-267.

Çoban, D., Suzer, C., Kamaci, H. O., Saka, Ş., \& Firat, K. (2009). Early osteological development of the fins in the hatchery-reared red porgy, Pagrus pagrus (L. 1758). Journal of Applied Ichthyology, 25, 26-32.

Fernández, I., Hontoria, F., Ortiz-Delgado, J. B., Kotzamanis, Y., Estévez, A., Zambonino-Infante, J. L., \& Gisbert, E. (2008). Larval performance and skeletal deformities in farmed gilthead sea bream (Sparus aurata) fed with graded levels of vitamin A enriched rotifers (Brachionus plicatilis). Aquaculture, 283(1), 102-115.

Fischer, W., Krupp, F., Schneider, W., Sommer, C., Carpenter, K. E., \& Niem, V. (1995). Guía FAO para identificación de especies para los fines de la pesca Pacífico centro-oriental. Volumen III. Vertebrados, Parte 2. Roma: FAO.

Fraser, M. R., Anderson, T. A., \& de Nys, R. (2004). Ontogenic development of spine and spinal deformities in larval barramundi (Lates calcarifer) culture. Aquaculture, 242(1), 697-711.

Gállego, L. (2008). Los cordados. Funciones de control voluntario. Vigo: Fundación Laboratorio de Biología Animal.

García-Ortega, A., Abdo-de la Parra, I., Duncan, N. J., Rodríguez-Ibarra, E., Velasco-Blanco, G., González-Rodríguez, B., Puello-Cruz, A., \& Martínez, I. (2005). Larval rearing of spotted rose snapper Lutjanus guttatus under experimental conditions. In C. I. Hendry, G. Van Stappen, M. Willie, \& P. Sorgeloos (Eds.), Larvi 05. Fish and Shellfish Larviculture Symposium (36, 172-175). European Aquaculture Society, Special Publication.

García-Ortega, A. (2007). Estado actual de la investigación y producción de botete diana Sphoeroides annulatus. 
In M. L. González-Félix, L. Bringas-Alvarado, M. Pérez-Velázquez, \& M. Meza-García (Eds.), Memorias del 3er Foro Internacional de Acuicultura (pp. 58-83). México.

Gavaia, P. J., Dinis, M. T., \& Cancela, M. L. (2002). Osteological development and abnormalities of vertebral column and caudal skeleton in larval and juvenile stages of hatchery-reared Senegal sole (Solea senegalensis). Aquaculture, 211, 305-323.

Hernández, D. R., Santinón, J. J., Sánchez, S., \& Domitrovic, H. A. (2013). Crecimiento, supervivencia e incidencia de malformaciones óseas en distintos biotipos de Rhamdia quelen durante la larvicultura. Latin American Journal of Aquatic Research, 41(5), 877-887.

Ibarra-Castro, L., \& Álvarez-Lajonchère, L. S. (2011). GnRHa-induced multiple spawns and volition spawning of captive spotted rose snapper, Lutjanus guttatus, at Mazatlán, México. Journal of the World Aquaculture Society, 42(4), 564-574.

Koumoundouros, G., Gagliardi, F., Divanach, P., Boglione, C., Cataudella, S., \& Kentouri, M. (1997). Normal and abnormal osteological development of caudal fin in Sparus aurata L. fry. Aquaculture, 149, 215-226.

Koumoundouros, G., Divanach, P., \& Kentouri, M. (1999). Osteological development of the vertebral column and of the caudal complex in Dentex dentex. Journal of Fish Biology, 54, 424-436.

Koumoundouros, G., Divanach, P., \& Kentouri, M. (2001a). Osteological development of Dentex dentex (Osteichthyes: Sparidae): dorsal, anal, paired fins and squamation. Marine Biology, 138, 399-406.

Koumoundouros, G., Sfakianakis, D. G., Maingot, E., Divanach, P., \& Kentouri, M. (2001b). Osteological development of the vertebral column and of the fins in Diplodus sargus (Teleostei: Perciformes: Sparidae). Marine Biology, 139, 853-862.

Laggis, A., Sfakianakis, D. G., Divanach, P., \& Kentouri, M. (2010). Ontogeny of the body skeleton in Seriola dumerili (Risso, 1810). Italian Journal of Zoology, 77(3), 303-315.
Lagler, K. F., Bardach, J. E., Miller, R. R., \& May Passino, D. R. (1990). Ictiología (2da. Ed.). México: AGT Editor.

Lewis-McCrea, L. M., Lall, S. P., \& Eckhard, W. P. (2004). Morphological descriptions of the early stages of spine and vertebral development in hatchery-reared larval and juvenile Atlantic halibut (Hippoglossus hippoglossus). Aquaculture, 241, 47-59.

Meunier, F. J., \& Ramzu, M. Y. (2006). La regionalization morphofonctionnelle de l'axe vertebral chez les Teléostéens en relation avec le mode de nage. Comptes Rendus Palevol, 5, 499-507.

Potthoff, T. (1984). Clearing and staining techniques. In H. G. Moser, W. J. Richards, D. M. Cohen, M. P. Fahay, A. W. Kendall Jr., \& S. L. Richardson (Eds.), Ontogeny and Systematics of Fishes (pp. 35-37). American Society of Ichthyologists and Herpetologists. Special Publication. No. 1. Lawrence: Allen Press.

Rodríguez-Ibarra, L. E., Aguilar-Zarate, G., Velasco-Blanco, G., \& Ibarra-Castro, L. (2013a). Tinción de tejidos óseos y cartilaginosos del pargo lunarejo Lutjanus guttatus. Industria Acuícola, 9, 10-13.

Rodríguez-Ibarra, L. E., Abdo-de la Parra, M. I., VelascoBlanco, G., González-Rodríguez, B. T., DomínguezJiménez, V. P., García-Aguilar, N., \& Ibarra-Castro, L. (2013b). Efecto de la eliminación de la capa adherente de los huevos utilizando enzima proteolítica proteasa y jugo de piña en la larvicultura del botete diana Sphoeroides annulatus. Revista de Biología Marina y Oceanografia, 48, 379-385.

Steindachner, F. 1869. Ichthyologische Notizen. Sitzber. Akad. Wiss. Wien, 60, 290-318.

Thomson, D. A., Findley, L. T., \& Kerstitch, A. N. (2000). Reef fishes of the sea of Cortez. The rocky-shore fishes of the golf of California (3ra. ed.). Arizona: The University of Arizona Press.

Wagemans, F., Focant, B., \& Vandewalle, P. (1998). Early development of the cephalic skeleton in the turbot. Journal of Fish Biology, 52, 166-204.

Wagemans, F., \& Vandewalle, P. (1999). Development of the cartilaginous skull in Solea solea: trends in Pleuronectiforms. Annales des Sciences Naturelles Zoologie et Biologie Animale, 20(1), 39-52. 